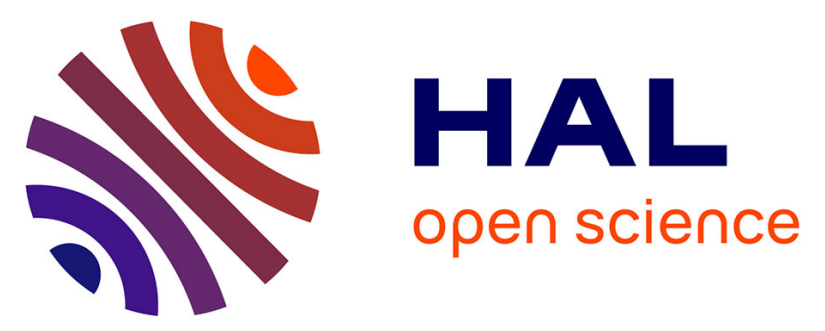

\title{
CALCULATION OF THE ELASTIC AND ELECTROSTATIC ENERGIES OF AN INCLUSION IN A FERROELECTRIC OR A PIEZOELECTRIC CRYSTAL
}

\author{
M. Vallade, G. Dolino
}

\section{To cite this version:}

M. Vallade, G. Dolino. CALCULATION OF THE ELASTIC AND ELECTROSTATIC ENERGIES OF AN INCLUSION IN A FERROELECTRIC OR A PIEZOELECTRIC CRYSTAL. Journal de Physique Colloques, 1982, 43 (C4), pp.C4-401-C4-404. 10.1051/jphyscol:1982459 . jpa-00222177

\section{HAL Id: jpa-00222177 https://hal.science/jpa-00222177}

Submitted on 1 Jan 1982

HAL is a multi-disciplinary open access archive for the deposit and dissemination of scientific research documents, whether they are published or not. The documents may come from teaching and research institutions in France or abroad, or from public or private research centers.
L'archive ouverte pluridisciplinaire HAL, est destinée au dépôt et à la diffusion de documents scientifiques de niveau recherche, publiés ou non, émanant des établissements d'enseignement et de recherche français ou étrangers, des laboratoires publics ou privés. 
JOURNAL DE PHYSIQUE

Colloque C4, supplément ou $n^{\circ}$ 12, Tome 43, décembre 1982

page $\mathrm{C4-401}$

\title{
CALCULATION OF THE ELASTIC AND ELECTROSTATIC ENERGIES OF AN INCLUSION IN A FERROELECTRIC OR A PIEZOELECTRIC CRYSTAL
}

\author{
M. Vallade and G. Dolino
}

Laboratoire de Spectrométrie Physique de l'Université Scientifique et Médicale de Grenoble, associé ou C.N.R.S. (L.A. no 8), B.P. S3X, 38041 Grenoble Cedex, France

(Revised text accepted 5 October 1982)

\begin{abstract}
Previous optical observations have shown that the first order structural phase transitions of some ferroelectric (e.g. $\mathrm{KD}_{2} \mathrm{PO}_{4}$ ) or piezoelectric (e.g. quartz) crystals present some similarity with martensitic transformations : two phases with different lattice parameters coexist over a small temperature range and the low temperature phase can be twinned; (this twinning corresponds to alternate shears and electric polarization in the case of $\mathrm{KD} \mathrm{PO}_{4}$ ). In contrast with martensites both elastic and electric properties are important in determining the morphology of the heterophase structure. In particular the habit plane separating the two phases is no longer a plane without long range strains when electric energies are not negligible. In this case one has to determine the configuration for which the sum of the elastic and electric energies is minimum : this can be done for an inclusion of the new phase in an infinite matrix of the parent phase by extending Khachaturyan's theory to take into account the electric and piezecelectric properties. We present an outline of this theory in the case of crystals of any symmetry. The effect of a uniform applied stress or of an electric field is also discussed.
\end{abstract}

Introduction.- Many insulating crystals exhibit a structural phase transition which is often found to be first order (1). In these cases there is a small temperature range corresponding to the coexistence of a stable and of a metastable phase. Interesting problems are associated with the properties of this mixed state : morphology of the heterophase structure, orientations and dynamics of phase boundaries. This situation is rather similar to that encountered during thermoelastic martensitic phase transformations. However, while these problems have been thoroughly investigated in metals, much less attention has been paid to the case of insulating crystals : this is due to the fact that the temperature range of phase coexistence is usually much smaller than for martensites (a few degrees or less). Some studies have been reported for ferroelectric crystals (2) and other insulating materials, such as $\mathrm{Zr} \mathrm{O}_{2}$ (3). Insulating crystals offer the advantage to be transparent in many cases, which allows simple optical microscopic investigations of bulk properties. Recently such experiments have been performed in our laboratory for piezoelectric crystals (quartz, $\mathrm{NH} \mathrm{Cl}$ ) and ferroelectric crystals $\left(\mathrm{KD}_{2} \mathrm{PO}_{4}\right)(4,5)$. Optical microscopy, however, is limited to a spatial resolution of a $f^{2}{ }^{4}$ microns and does not give any information about the first stages of the nucleation process. On the other hand it is well adapted to the study of heterophase structures, with a characteristic size in the $0.01-1 \mathrm{~mm}$ range, determined by long range interactions. In some cases, as in quartz, the morphology of the heterophase structure is essentially determined by the anisotropy of the properties of the crystal and is sensitive to an applied uniaxial stress (4). In ferroelectricferroelastic materials such as $\mathrm{KD}_{2} \mathrm{PO}_{4}$, a spontaneous polarization and a spontaneous shear strain appear in the low temperature phase, in addition to dilatational strains. Twins corresponding to opposite values of polarization and shear are systematically observed. If one could neglect the electrostatic energy, the habit plane between the two phases would be a plane without long range stresses as in the theory of martensites (6) (if such a plane is allowed by transformation strains). However this corresponds to an unequal proportion of the two kinds of twins and so to a net polarization 
to the boundary. In this case there are no simple geometrical considerations and one has to calculate the balance between elastic and electrostatic energies. As a first step, we propose an extension of Khachaturyan's theory (7) by taking into account electric and elastic properties in a similar way. Here we give only an outline of the theory (the detailed calculations, which are rather long, will be published elsewhere).

Energy of an inclusion in a infinite piezoelectric or ferroelectric crystal. - Let us consider an inclusion of arbitrary shape in an infinite matrix of arbitrary crystallographic symmetry. First, we assume that the elastic, dielectric and piezoelectric constants are the same in the inclusion and in the matrix. Due to their similar long range character, electric and elastic properties can be treated in a similar way :

- Electric energy : let us consider an inclusion with an electric induction $\vec{D}$, which in the absence of the matrix, would be uniform and equal to $\vec{D}_{0}$. The free energy density can be written (8) :

$$
\text { f }(\vec{r})=\frac{1}{2}\left[\left(\vec{D}-\vec{D}_{0}\right) A \cdot\left(\vec{D}-\vec{D}_{0}\right) \Theta(\vec{r})+\vec{D} \cdot A \cdot \vec{D}(1-\theta(\vec{r}))\right]
$$

where $\theta(\vec{r})$ is the form function of the inclusion $(\theta(\vec{r})=1$ inside, $\theta(\vec{r})=0$ outside). $A$ is the inverse of the permittivity tensor divided by $4 \pi$. In this paper the notation for matrix will be heavy character such as $A$. The electric fiela $\vec{E}=4 \pi \partial f / \partial D$

and $\vec{D}$ verify the electrostatic equation $\vec{\nabla} \times \vec{E}=0$ and $\vec{\nabla} \cdot \vec{D}=0$. Using Fourier transform, one obtains :

$$
\vec{D}(\vec{q})=F\left(\vec{q}_{u}\right) \cdot \vec{D}_{0} \theta(\vec{q})
$$

where $\boldsymbol{F}(\vec{q})$ is a non-symmetric, dimensionless 3 x 3 matrix depending only upon $A$ and the unit vector $\vec{q}$ parallel to $\vec{q}$. The matrix $\vec{f}$ is such that $F^{2}=F$ and F.A.F. = A.P. Its eigenvalues are $0,1,1$, which correspond to eigenvectors, respectively, longitudinal and transversal relative to $\vec{q}$. The mean value $\langle\vec{D}\rangle$ of $\vec{D}$ inģide the inclusion is given by $\langle\vec{D}\rangle=\langle\vec{p}\rangle$. $\vec{D}_{0}$, where $\langle\vec{p}\rangle$ is the average of $F(\vec{q})$ in $q$ space, weighted by the probability density $|\Theta(\vec{q})|^{2} / v^{2}$, where $v$ is the volume of the inclusion. The electrostatic energy $e_{1}$ is :

$$
e_{1}=\frac{1}{2} v\left(\vec{D}_{0} \cdot A \cdot\left(\vec{D}_{0}-\langle\vec{D}\rangle\right)\right)
$$

Elastic energy : Khachaturyan's theory can be written using a similar formalism. Letting $\vec{E}$ be the 6 components transformation strain vector, the elastic fxee energy is :

$$
f_{2}(\vec{r})=\frac{1}{2}\left[\left(\vec{\varepsilon}-\vec{\varepsilon}_{0}\right) \cdot \boldsymbol{c} \cdot\left(\vec{\varepsilon}-\vec{\varepsilon}_{0}\right) \theta(\vec{r})+\varepsilon \cdot \boldsymbol{c} \cdot \varepsilon(1-\theta(\vec{r})]\right.
$$

Where $\mathbf{c}$ is the elastic constant tensor. The stress $\vec{\sigma}=\frac{\partial f_{2}}{\partial \vec{\varepsilon}}$ verifies the equation
$\vec{\nabla} \cdot \vec{\sigma}=0$. Then :

$$
\vec{E}(\vec{q})=s\left(\vec{q}_{u}\right) \cdot \vec{\varepsilon}_{0} \Theta(\vec{q})
$$

$\mathbf{s}(\vec{q})$ is a nonsymmetric, dimensionless $6 \times 6$ matrix which depends only on the elastic constants and on the direction of $q$. It has the same properties as $\mathbf{F}\left(\mathbf{s}^{2}=\mathbf{s}\right.$ and $\left.\mathbf{s} . \mathbf{C . s .}=\mathbf{C . s}\right)$. The mean value of the strain in the inclusion is $\langle\vec{E}\rangle=\langle\mathbf{s}\rangle \cdot \vec{\varepsilon}_{0}$ and the elastic energy is :

$$
e_{2}=\frac{1}{2} \mathrm{v}\left(\vec{\varepsilon}_{0} \cdot C \cdot\left(\vec{\varepsilon}_{0}-\langle\vec{\varepsilon}\rangle\right)\right.
$$

The last term corresponds to the accomodation of the matrix. 
- Piezoelectric coupling : The previous formulation allows a straghtforward generalization to the case of piezoelectric crystals, for which there is a bilinear coupling between $\vec{D}$ and $\vec{\varepsilon}$. One can define a 9 components vector $\vec{x}=(\vec{D}, \vec{\varepsilon})$ and a $9 \times 9$ matrix $K=\left[\begin{array}{ll}A & H \\ H & C\end{array}\right]$ where $\boldsymbol{H}$ is the piezeoelectric stress tensor. Equilibrium equations lead to :

$$
\vec{x}(\vec{q})=v\left(\vec{q}_{u}\right) \cdot \vec{x}_{0} \theta(\vec{q})
$$

where the matrix $\mathbf{V}\left(\vec{q}_{U}\right)$ is a function of $A, \boldsymbol{H}, \mathbf{C}$ and $\vec{q}_{u}$. Once again $\boldsymbol{v}^{2}=\mathbf{v}$. The total energy (electrostatic, elastic and piezoelectric) is :

$$
e_{3}=\frac{1}{2} v \vec{x}_{0} \cdot x \cdot\left(\vec{x}_{0}-\langle\vec{x}\rangle\right)
$$

- Ellipsoidal inclusions :Then, the induction $\vec{D}$ and the strain $\vec{\varepsilon}$ are uniform inside the inclusion. In this case one can find a solution even when $C, A$ and $H$ take different values in the inclusion and in the matrix.

$$
f_{3}=\frac{1}{2}\left[\left(\vec{x}-\vec{x}_{0}\right) \cdot x_{i} \cdot\left(\vec{x}-\vec{x}_{0}\right) \theta(\vec{r})+\vec{x} \cdot x_{m} \cdot \vec{x}(1-\theta(\vec{r}))\right]
$$

The generalised stress field $\vec{x}$ is :

$$
\vec{x}=\partial f_{3} / \partial \vec{x}=-\boldsymbol{k}_{i} \cdot\left(\vec{x}_{0}-\mathbf{B} \cdot \vec{x}\right) \theta(\vec{r})+\boldsymbol{k}_{m} \cdot \vec{x}
$$

Where $\mathbf{z}=\mathbf{A}-\left(\mathbf{k}_{i}\right)^{-1} \cdot \mathbf{k}_{\mathrm{m}}$. Since $\vec{x}$ is uniform inside the inclusion, one may substitute $\langle\vec{x}\rangle$ and the problem is formally identical to that dexived previously. The solution is obtained from the self-consistency equation relating $\langle\vec{x} \cdot$ to $\vec{x}-\vec{B} .\langle\vec{x}\rangle$. This result generalises Eshelby's theory (9). In the case cf a non ellipsoidal inclusion, the above calculation corresponds to a mean-field approximation.

Applied stress and electric field : An exact solution can also be obtained for an ellipsoidal inclusion, undegx a uniform applied stress or electric field. The coupling

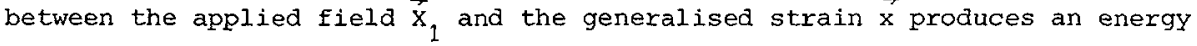

$$
e_{4}=\frac{1}{2} v \vec{x}_{1} \cdot\left(\vec{x}_{0}-\vec{B} \cdot\left(\vec{x}_{0}-\langle\vec{x}>)\right)\right.
$$

This energy does not depend on the inclusion shape if : $B=0$ (i.e. $\mathbf{x}_{i}=\mathbf{x}_{m}$ )

Discussion : These results allow a determination of the shape and orientation of an inclusion by minimizing the total electric and elastic energy. Such a simple model corresponds only to a rough description as several simplifying assumptions have been made :

- The sample is considered as infinite with only one inclusion. It is clear that for long range interactions the finite size and the shape of the sample play an important role. Furthermore the sample boundary is often the most favourable site for nucleation, the heterophase structure may consist only of two regions separated by a single continuous phase boundary ending on the crystal surfaces.

- The interfacial energy must be negligible. This can be valid only if no interfacial. dislocations are present (coherent interface) and if the inclusion is sufficiently large so that the ratio of surface energy to the volume energy is small.

- The model does not explicitly take into account the twinning of the low temperature phase. Then the spontaneous generalised strain $\vec{x}$ has to be considered as an average over the twin structure. One is thus led to make a distinction between the components of $\vec{x}_{0}$ which change their sign from one twin to the other and components which are invariant with twinning. The former ones may have a small. average value and the corresponding energy is decreased by twinning. 
For example in a ferroelectric-ferroelastic crystal 1 ike $\mathrm{KD}_{2} \mathrm{PO}_{4}$, the spontaneous shear $\varepsilon_{6}^{0}$ and the spontaneous polarization $D_{3}^{\circ}$ are proportiontal to the order parameter and they take alternate values in different twins. On the other hand, the dilatation strain components $\varepsilon_{1}^{\circ}=\varepsilon^{\circ}$ and $\varepsilon_{0}^{\circ}$ are proportionnal to the square of the order parameter, due to spontaneous electrostriction. In general without external fields there is a periodic twinned structure (10). As in $\mathrm{KD}_{2} \mathrm{PO}_{4}, \varepsilon_{1}^{0}$ and $\varepsilon_{3}^{\circ}$ are both positive (4), no invariant plane can exist and the twinning corresponds to a nearly equal proportion of each kind of twins so that $\left\langle\varepsilon_{6}^{0}\right\rangle\left\langle D_{3}^{\circ}\right\rangle=0$. The orientation of the phase boundary is then essentially determined by the relative values of $\varepsilon_{1}^{0}$ and $\varepsilon_{3}^{0}$. When a d.c. electric field $E_{3}$ is applied the twinning disappears and the energy associated to $\varepsilon_{6}^{\circ}$ and $D_{3}^{\circ}$ becomes predominant. This explains the drastic changes observed as a function of the field (5).

In conclusion, investigation of first order stuctural phase transition in insulating crystal may provide new illustrations of martensitic-like heterophase structure. Furthermore, some new features related to ferroelectricity and piezoelectricity can lead to an extension of these theories. The sensitivity of these materials to applied stresses or electric fields makes investigation of the dynamics properties of phase boundaries and twin walls possible.

\section{REFERENCES}

1) BRUCE A.D. and COWLEY R.A., "Structural phase transitions", Taylor and Francis Ltd, London (1981).

2) DI DOMENICO M. and WEMPLE S.H., Phys. Rev 155 (1967) 539.

3) KRIVEN W.M.Proceedings of the Internat. Conf. on Solid-Solid phase transformations, Pittsburgh, U.S.A. 1981. (to be published).

4) BACHHEIMER J.P., BASTIE P., BORNAREI, J., DOLINO G. and VALLADE M., Proceedj.ngs _of the Internat. Conf on Solid-Solid phase transformations, Pittusburg, U.S.A. (1981) (to be published).

5) BASTIE P., BORNAREL J., DOLINO G., and VALLADE M., Ferroelectrics, 26 (1980) 789.

6) CHRISTIAN J.W., "The Theory of transformations in Metals and Alloys" Pergamon Press, Inc, New-York (1965).

7) KHACHATURYAN A.G., Soviet Phys. So1. State $\underline{8}$ (1967) 2131.

8) LANDAU L. and LIFCHITZ E., "Electrodynamique des milieux continus" Ed. Mir, Moscow 1969, page 86.

9) ESHELBY J.D., Proc.Roy.Soc. A 241 (1957) 376.

10) BORNAREL J., and BASTIE P., J. Phys. C : Solid St.Phys. 13 (1980) 5843. 